\title{
8. LEG 2 GRAIN SIZE ANALYSIS AND COMPOSITION OF THE COARSE FRACTION
}

\author{
Deep Sea Drilling Project East Coast Repository \\ Lamont-Doherty Geological Observatory, Palisades, N. Y.
}

\begin{abstract}
Methods used for the grain size analyses were described in the preliminary core descriptions for Leg 1 . A complete list of the results of the grain size analyses is given as Table 1, and a summary of the composition of the coarse fraction is given as Table 3. Owing to the damaging effects of the ultrasonic probe used to disperse the sediments, many of the more delicate microfossils that would normally form part of the sand fraction may have been broken so that they now form part of the silt fraction, thus biasing the percentage of the latter.
\end{abstract}

\section{Site 8}

In Hole 8, Core 1 and most of Core 2 are comprised of sediment in the silty clay size range and this is mainly mud and radiolarian ooze with some fine turbidites. Towards the base of Core 2, the sediment becomes slightly coarser, clayey silt and forms a spiculate silt.

The sand fraction in Holes 8 and $8 \mathrm{~A}$ varies from 0.5 to 5.2 per cent and is composed mainly of Radiolaria and sponge spicules.

\section{Site 9}

Nearly all of the cores from Hole 9 are of clay, with two exceptions, these being silty clay layers in Core 5 . Some of the clays are very pure-containing as much as 98 per cent clay; the average is 88.6 per cent clay. Although all of these sediments fall in the clay size range, there is a noticeable decrease in the silt content (sand is almost negligible) from Core 7 down. In Core 5 the silt content is about 20 per cent; but, in Cores 7 through 12 , the silt content is around 10 per cent or less. This change in the silt content is reflected in the nature of sediments which are comprised of nannofossil oozes above and pass into zeolitic clays below.

The sand fraction in Core 5 of Hole 9 is less than 1 per cent, and mainly consists of Radiolaria, some sponge spicules, varying amounts of foraminifera and rare manganese and glass shards.

Cores 7 through 12 usually contain less than 1 per cent sand which mainly comprises fish debris, some Radiolaria and foraminifera fragments and less commonly manganese and glass shards.

In Sections 5 and 6 of Core 10 an unusual and distinctive mineral makes up almost the entire sand fraction
In thin section the mineral forms colorless elongate crystals which are often grouped into rosettes or crossshaped clusters (rather like penetration twins). Under high power it can be seen that these elongate crystals are in fact composite crystals, the whole unit having a serrated margin formed by minute crystal faces. This mineral has a relief slightly less than balsam, and a low birefringence (1st order yellow and white), and shows wavy extinction parallel to the long axis. A photomicrograph of this mineral is given as Plate 1A. A small amount of the mineral was ground up and the powder puddled in water (so the mineral would take up any preferred orientation) and run on the X-ray diffractometer. ${ }^{1}$ The mineral gave a multiple main peak at $2.81 \AA$ and $2.84 \AA$, with minor peaks at $1.77 \AA$ and $1.74 \AA$. Therefore on the basis of the X-ray diffraction data the mineral would appear to be a phosphate, probably one of the apatites (hydroxlapatite, chlorapatite, or flourapatite). Section 6 of Core 8 contained a coarse fraction entirely composed of tubes (burrow infills or faecal pellet origin?) of a carbonate mineral-probably rhodochrosite. ${ }^{2}$

Hole 9A Core 1 is comprised of zeolitic clays with a silt content of 6 to 12 per cent; the sample from Section 5 in this core contains 12.9 per cent sand made up predominantly of chert fragments varying in size from 0.05 to 5 millimeters. The other two samples from Core 1 contain less than 1 per cent sand consisting predominantly of rhodochrosite, with rare manganese and fish debris. Core 3 of Hole 9A contains 18 to 28 per cent silt, but it is also a zeolitic clay or silty clay. Possibly the relatively high content of silt for a zeolitic sediment is due to the abundance of radiolarian fragments. The sand fraction in Core 3 varies from 0.5 to 1.1 per cent and comprises mainly Radiolaria and rhodochrosite in Sections 2 and 4; a photomicrograph of rhodochrosite in Section 4 is given as Plate 1B. Sections 1 and 4 however, also contain an abundance of the zeolite mineral clinoptilolite (identified by X-ray diffraction). A photomicrograph of this mineral is given as Plate 1C. Clinoptilolite in Core 3, Section 1 forms clusters of small colorless elongate crystals; the crystal

X-ray identifications made by Marion Jacobs, Lamont-Doherty ,Geological Observatory.

All identifications of rhodochrosite were originally considered by the author to be dolomite, but he understands that this is not so on Rex's X-ray work. (Chapter 11). 
have a low relief (less than balsam) and a very weak birefringence (1st order whites) and show extinction parallel to the long direction of the crystals i.e., 010. Clinoptilolite was also common in the sand fraction of Section 5, Core 1.

\section{Site 10}

Sediments from Hole 10 are mainly silty clays or, less commonly, clays formed from nannofossil chalk or marl oozes; rarely are clayey silts present when the nannofossil content is particularly high. Some zeolitic clay occurs admixed with the oozes in the upper part of the hole. Sand is almost negligible in the upper part of Hole 10 , but increases to about 3 per cent lower down. The composition of the coarse fraction is given in Tables 2 and 3 . It can be seen from the tables that foraminifera and smaller amounts of fish fragments are the main constituents. It will also be seen that in Cores $2,3,5$ and 7 varying amounts of what is tentatively identified as clinoptilolite also occur in the sand fraction. However, clinoptilolite in this hole has a brown coating which partially masks the characters of the mineral; in some places traces of the crystal form seen in this mineral in Hole 9 are still visible (Plate 1D).

\section{Site 11}

Core 1 of Hole 11 comprises clay with only 8 per cent silt in its upper part, with silty clay in Sections 2 through 4 . The sediment in this core, is a nannofossil foraminifera chalk ooze. The sand content in Hole 11 is high compared with other sites from Leg 2 and varies from 8 to 14 per cent. It consists entirely of planktonic foraminifera.

Hole 11A contains a high proportion of silt grade material-the sediment varying from clayey silt to silt. The unusually high silt content is due to the abundance of nannofossils in this nannofossil chalk ooze. The sand fraction forms about 2 per cent of the sediment and consists of planktonic foraminifera.

\section{Site 12}

All sediments from Hole 12B were of zeolitic clay with clay grade material forming as much as 96 per cent of the sediment in places. The sand fraction comprised less than 1 per cent of the sediment and consisted entirely of volcanic glass in $12 \mathrm{~B}, 1-4$, and predominantly manganese with some glass and Radiolaria in 12B, 2-2.

In Hole $12 \mathrm{C}$, Core 1 sediments range from clayey silt to silty clay-the silt content decreasing downwards from 74 to 37 per cent; the sediment here is a nannofossil foraminifera chalk ooze. The sand in Core 1 of Hole $12 \mathrm{C}$ decreases from 11.6 to 8.5 per cent lower down, and consists predominantly of foraminifera with rare glass and manganese.

Core $2 \mathrm{R}$ in Hole $12 \mathrm{C}$ passes from a silty clay down into clay grade material. The sediment here again is a nannofossil foraminifera chalk ooze. The sand percentage varies from 0.5 to 5.0 depending on the amount of foraminifera present. Core 4 is the same sediment and falls in the silty grade size. The coarse fraction is mainly foraminifera, with some rare fish and glass fragments.

Cores 5 through 11 are mostly clay showing a progressive decrease in silt to less than 5 per cent at the bottom of Core 11. The sediment in these cores is zeolitic. In Core 5 the sand fraction is mainly formed of small clusters of minute ?clinoptilolite crystals which are coated with a pale brown-material; some manganese, glass and fish fragments also occur. In Core 6 manganese is the predominant mineral in the sand fraction, and in Cores 8 and 9 glass fragments are most common. Clusters of brown-coated ?clinoptilolite (similar to that in Core 5) occur rarely in Cores 6 and 8 .

\section{PLATE 1 \\ Minerals from samples taken on Leg 2.}

1 A ?Apatite variety. (Sample 2-9-10-6, 10-12. cm). PPL

1B Rhodochrosite (Sample 2-9A-3-4, $134 \mathrm{~cm}$ ) $\times$ 80. PPL

1C Clinoptilolite (Sample 2-9A-3-1, 82-84 cm) X 80. PPL

1D Clinoptilolite coated with pale brown mineral. (Sample $2-10-2-3,99-101 \mathrm{~cm}) \times 80 . \mathrm{PPL}$ 

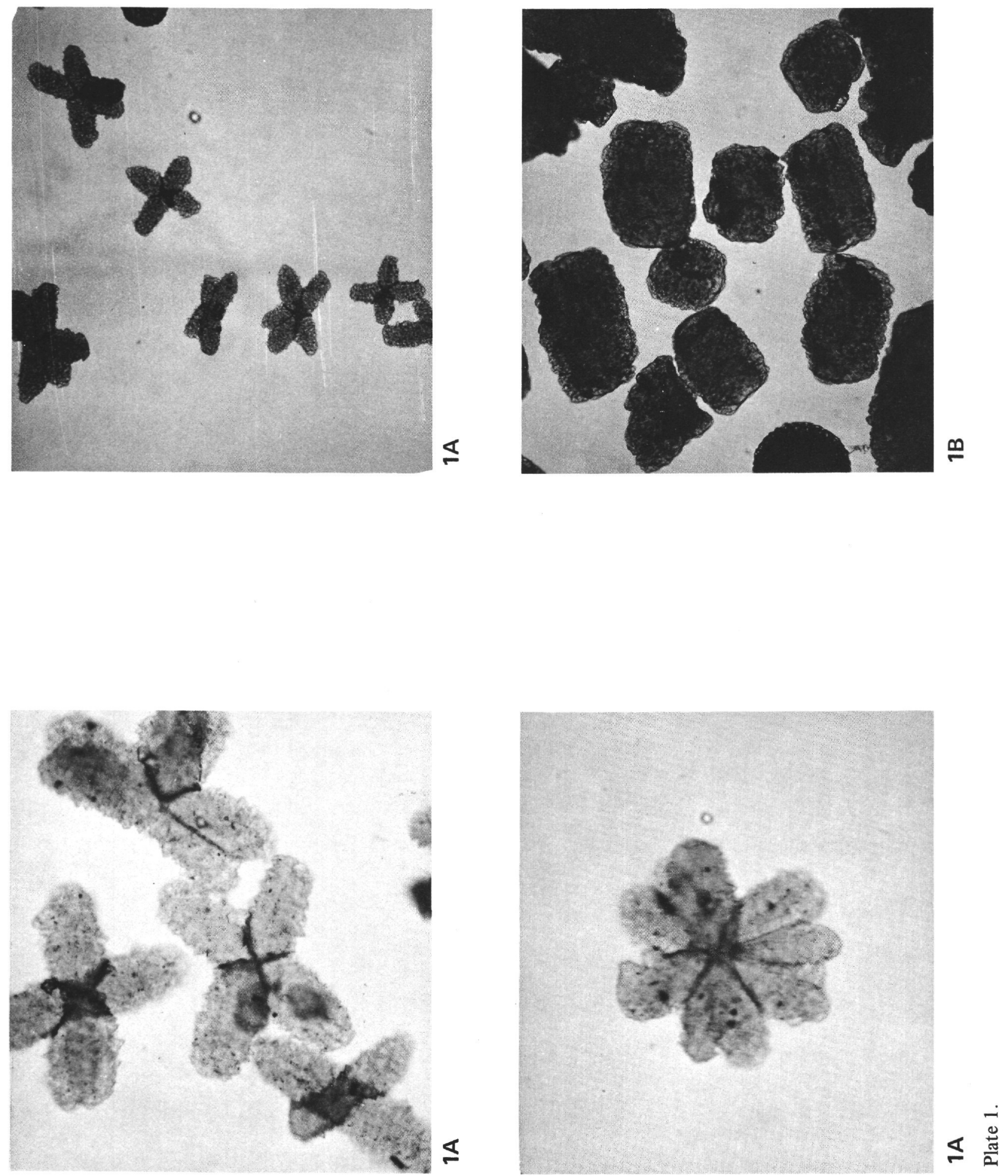

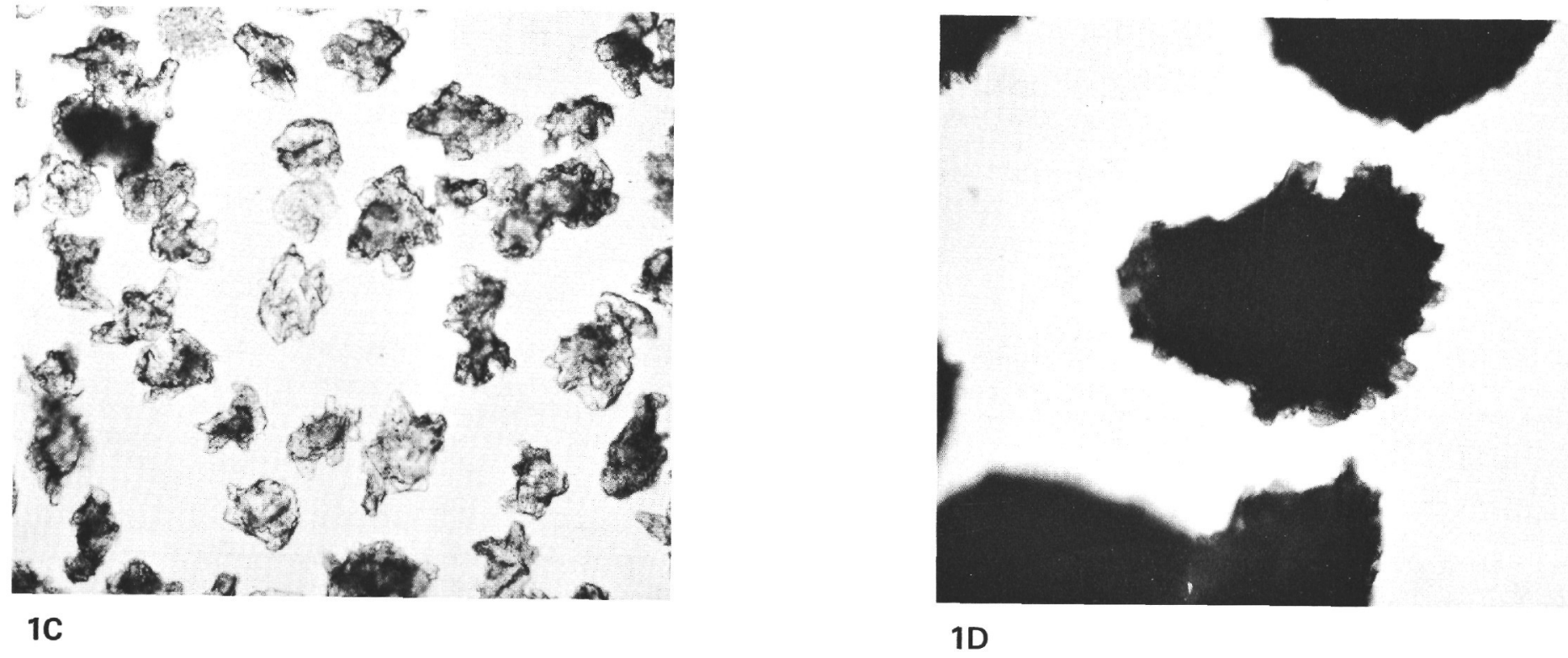

9

Plate 1. Continued. 
TABLE 1

Leg 2 Grain Size Results

\begin{tabular}{|c|c|c|c|c|c|c|c|}
\hline Hole & Core & Section & Sampled & $\%$ Sand & $\%$ Silt & $\%$ Clay & Classification \\
\hline 8 & 1 & 1 & $60-62$ & 0.5 & 48.9 & 50.6 & Silty clay \\
\hline 8 & 2 & 1 & $100-102$ & 4.4 & 34.2 & 61.4 & Silty clay \\
\hline 8 & 2 & 2 & $6-8$ & 3.3 & 45.7 & 51.0 & Silty clay \\
\hline 8 & 2 & 3 & $12-14$ & 4.9 & 30.5 & 64.6 & Silty clay \\
\hline 8 & 2 & 3 & $72-74$ & 5.2 & 61.0 & 33.8 & Clayey silt \\
\hline $8 \mathrm{~A}$ & 1 & 1 & $102-104$ & 2.0 & 68.2 & 29.8 & Clayey silt \\
\hline $8 \mathrm{~A}$ & 1 & 2 & $6-8$ & 0.4 & 63.6 & 36.0 & Clayey silt \\
\hline 9 & 5 & 1 & $14-16$ & 0.3 & 25.6 & 74.1 & Silty clay \\
\hline 9 & 5 & 1 & $82-84$ & 0.2 & 19.6 & 80.2 & Clay \\
\hline 9 & 5 & 2 & $12-14$ & 0.0 & 21.2 & 78.8 & Clay \\
\hline 9 & 5 & 2 & $88-90$ & 0.1 & 16.3 & 83.6 & Clay \\
\hline 9 & 5 & 3 & $6-7$ & 0.1 & 20.0 & 79.9 & Clay \\
\hline 9 & 5 & 4 & $7-9$ & 0.3 & 21.3 & 78.4 & Clay \\
\hline 9 & 5 & 4 & $82-84$ & 0.6 & 36.8 & 62.6 & Silty clay \\
\hline 9 & 5 & 5 & $7-9$ & 0.0 & 20.4 & 79.6 & Clay \\
\hline 9 & 5 & 5 & $83-85$ & 0.6 & 22.3 & 77.1 & Clay \\
\hline 9 & 7 & 1 & $126-128$ & 0.0 & 10.6 & 89.4 & Clay \\
\hline 9 & 7 & 2 & $90-92$ & 0.1 & 11.3 & 88.6 & Clay \\
\hline 9 & 7 & 4 & $74-76$ & 0.0 & 11.5 & 88.5 & Clay \\
\hline 9 & 8 & 4 & $100-102$ & 0.0 & 9.4 & 90.6 & Clay \\
\hline 9 & 8 & 5 & $15-18$ & 0.0 & 11.1 & 88.9 & Clay \\
\hline 9 & 8 & 6 & $18-22$ & 1.4 & 9.6 & 89.0 & Clay \\
\hline 9 & 9 & 1 & $86-88$ & 0.0 & 8.5 & 91.5 & Clay \\
\hline 9 & 9 & 2 & $3-5$ & 0.0 & 10.4 & 89.6 & Clay \\
\hline 9 & 9 & 2 & $78-80$ & 0.1 & 6.8 & 93.1 & Clay \\
\hline 9 & 9 & 3 & $7-10$ & 0.0 & 2.0 & 98.0 & Clay \\
\hline 9 & 10 & 1 & $100-103$ & 0.1 & 1.8 & 98.1 & Clay \\
\hline 9 & 10 & 2 & $80-83$ & 0.1 & 6.5 & 93.4 & Clay \\
\hline 9 & 10 & 3 & $5-7$ & 0.1 & 5.4 & 94.5 & Clay \\
\hline 9 & 10 & 3 & $74-76$ & 0.0 & 8.3 & 91.7 & Clay \\
\hline 9 & 10 & 4 & $4-6$ & 0.0 & 7.6 & 92.4 & Clay \\
\hline 9 & 10 & 4 & $66-68$ & 0.0 & 7.2 & 92.8 & Clay \\
\hline 9 & 10 & 5 & $8-10$ & 0.1 & 9.5 & 90.4 & Clay \\
\hline 9 & 10 & 5 & $74-76$ & 0.0 & 10.6 & 89.4 & Clay \\
\hline 9 & 10 & 6 & $10-12$ & 0.2 & 7.9 & 91.9 & Clay \\
\hline 9 & 12 & 5 & $19-21$ & 0.1 & 6.4 & 93.5 & Clay \\
\hline $9 \mathrm{~A}$ & 1 & 1 & $3-5$ & 0.5 & 6.1 & 93.4 & Clay \\
\hline $9 \mathrm{~A}$ & 1 & 5 & $18-21$ & 12.9 & 12.3 & 74.8 & Sandy clay \\
\hline $9 A$ & 1 & 6 & $65-67$ & 0.7 & 10.7 & 88.6 & Clay \\
\hline
\end{tabular}


TABLE 1 - Continued

\begin{tabular}{|c|c|c|c|c|c|c|c|}
\hline Hole & Core & Section & Sampled & $\%$ Sand & $\%$ Silt & $\%$ Clay & Classification \\
\hline $9 \mathrm{~A}$ & 3 & 1 & $82-84$ & 0.5 & 18.8 & 80.7 & Clay \\
\hline $9 A$ & 3 & 2 & $65-68$ & 0.4 & 28.4 & 71.2 & Silty clay \\
\hline $9 \mathrm{~A}$ & 3 & 4 & 134 & 1.1 & 21.5 & 77.4 & Clay \\
\hline 10 & 1 & 1 & $60-62$ & 0.5 & 18.5 & 81.0 & Clay \\
\hline 10 & 1 & 2 & $13-15$ & 0.1 & 15.1 & 84.8 & Clay \\
\hline 10 & 2 & 2 & $12-14$ & 0.2 & 16.3 & 83.5 & Clay \\
\hline 10 & 2 & 2 & $129-131$ & 2.2 & 25.0 & 72.8 & Silty clay \\
\hline 10 & 2 & 3 & $13-15$ & 0.4 & 25.0 & 74.6 & Silty clay \\
\hline 10 & 2 & 3 & $99-101$ & 4.1 & 17.7 & 78.2 & Clay \\
\hline 10 & 2 & 4 & $5-7$ & 0.1 & 16.5 & 83.4 & Clay \\
\hline 10 & 3 & 1 & $35-37$ & 0.1 & 48.2 & 51.7 & Silty clay \\
\hline 10 & 3 & 2 & $16-18$ & 0.1 & 38.4 & 61.5 & Silty clay \\
\hline 10 & 3 & 3 & $10-12$ & 0.1 & 37.5 & 62.4 & Silty clay \\
\hline 10 & 3 & 4 & $12-14$ & 0.1 & 12.2 & 87.7 & Clay \\
\hline 10 & 3 & 4 & $125-127$ & 0.1 & 25.4 & 74.5 & Silty clay \\
\hline 10 & 3 & 3 & $81-83$ & 0.0 & 29.3 & 70.7 & Silty clay \\
\hline 10 & 5 & 1 & $118-120$ & 0.1 & 11.1 & 88.8 & Clay \\
\hline 10 & 5 & 2 & $12-14$ & 0.1 & 44.6 & 55.3 & Silty clay \\
\hline 10 & 5 & 2 & $74-76$ & 0.2 & 17.6 & 82.2 & Clay \\
\hline 10 & 5 & 3 & $18-20$ & 0.0 & 54.1 & 45.9 & Clayey silt \\
\hline 10 & 5 & 4 & $20-22$ & 0.1 & 43.6 & 56.3 & Silty clay \\
\hline 10 & 5 & 5 & $20-22$ & 0.1 & 30.4 & 69.5 & Silty clay \\
\hline 10 & 7 & 1 & $110-112$ & 0.1 & 47.9 & 52.0 & Silty clay \\
\hline 10 & 7 & 2 & $40-42$ & 0.1 & 43.6 & 56.3 & Silty clay \\
\hline 10 & 7 & 3 & $2-4$ & 0.1 & 37.9 & 62.0 & Silty clay \\
\hline 10 & 7 & 3 & $80-82$ & 0.1 & 43.2 & 56.7 & Silty clay \\
\hline 10 & 7 & 4 & $8-10$ & 0.2 & 34.3 & 65.5 & Silty clay \\
\hline 10 & 7 & 4 & $72-74$ & 0.1 & 30.2 & 69.7 & Silty clay \\
\hline 10 & 9 & 1 & $45-47$ & 4.9 & 24.0 & 71.1 & Silty clay \\
\hline 10 & 9 & 1 & $104-107$ & 3.5 & 52.8 & 43.7 & Clayey silt \\
\hline 10 & 9 & 2 & $20-22$ & 2.3 & 55.6 & 42.1 & Clayey silt \\
\hline 10 & 10 & 1 & $100-102$ & 2.5 & 37.0 & 60.5 & Silty clay \\
\hline 10 & 10 & 2 & $22-24$ & 2.3 & 66.0 & 31.7 & Clayey silt \\
\hline 10 & 10 & 2 & $84-86$ & 1.8 & 27.0 & 71.2 & Silty clay \\
\hline 10 & 10 & 3 & $10-12$ & 1.9 & 26.5 & 71.6 & Silty clay \\
\hline 10 & 10 & 3 & $80-82$ & 1.4 & 48.9 & 49.7 & Silty clay \\
\hline 10 & 10 & 4 & $13-15$ & 1.8 & 35.1 & 63.1 & Silty clay \\
\hline 10 & 10 & 4 & $82-84$ & 1.8 & 32.8 & 65.4 & Silty clay \\
\hline 10 & 10 & 5 & $14-16$ & 2.9 & 33.7 & 63.4 & Silty clay \\
\hline 10 & 10 & 5 & $72-74$ & 2.4 & 30.4 & 67.2 & Silty clay \\
\hline
\end{tabular}


TABLE 1 - Continued

\begin{tabular}{|c|c|c|c|c|c|c|c|}
\hline Hole & Core & Section & Sampled & $\%$ Sand & $\%$ Silt & $\%$ Clay & Classification \\
\hline 10 & 11 & 1 & $139-141$ & 3.4 & 32.5 & 64.1 & Silty clay \\
\hline 10 & 11 & 3 & $20-22$ & 4.0 & 31.4 & 64.6 & Silty clay \\
\hline 10 & 11 & 4 & $5-7$ & 4.2 & 30.2 & 65.6 & Silty clay \\
\hline 10 & 12 & 2 & $14-16$ & 2.9 & 28.3 & 68.8 & Silty clay \\
\hline 10 & 12 & 3 & 8-10 & 2.2 & 10.6 & 87.2 & Clay \\
\hline 10 & 12 & 3 & $68-70$ & 2.6 & 10.1 & 87.3 & Clay \\
\hline 10 & 12 & 4 & $12-14$ & 3.6 & 9.6 & 86.8 & Clay \\
\hline 10 & 12 & 4 & $75-77$ & 2.4 & 9.0 & 88.6 & Clay \\
\hline 10 & 13 & 1 & $120-122$ & 0.9 & 7.7 & 91.4 & Clay \\
\hline 10 & 13 & 2 & $5-7$ & 0.7 & 29.5 & 69.8 & Silty clay \\
\hline 10 & 13 & 3 & $15-17$ & 2.4 & 9.3 & 88.3 & Clay \\
\hline 10 & 13 & 4 & $10-12$ & 0.3 & 22.2 & 77.5 & Clay \\
\hline 10 & 14 & 2 & $12-14$ & 2.8 & 29.9 & 67.3 & Silty clay \\
\hline 10 & 14 & 3 & 8-10 & 0.9 & 27.6 & 71.5 & Silty clay \\
\hline 10 & 15 & 1 & $114-116$ & 2.6 & 33.8 & 63.6 & Silty clay \\
\hline 10 & 15 & 2 & 8-10 & 3.0 & 30.3 & 66.7 & Silty clay \\
\hline 10 & 15 & 2 & $83-85$ & 3.2 & 37.0 & 59.8 & Silty clay \\
\hline 10 & 16 & 1 & $185-187$ & 3.2 & 29.3 & 67.5 & Silty clay \\
\hline 10 & 16 & 2 & 4-6 & 3.0 & 32.0 & 65.0 & Silty clay \\
\hline 10 & 16 & 3 & $121-123$ & 2.2 & 8.1 & 89.7 & Clay \\
\hline 10 & 17 & 1 & $100-102$ & 2.7 & 28.0 & 69.3 & Silty clay \\
\hline 10 & 17 & 2 & $7-9$ & 2.9 & 28.3 & 68.8 & Silty clay \\
\hline 10 & 17 & 3 & $4-6$ & 2.1 & 13.2 & 84.7 & Clay \\
\hline 10 & 18 & 1 & $16-18$ & 2.4 & 24.0 & 73.6 & Silty clay \\
\hline 10 & 18 & 2 & $14-16$ & 2.4 & 36.4 & 61.2 & Silty clay \\
\hline 10 & 18 & 3 & $6-8$ & 1.2 & 31.0 & 67.8 & Silty clay \\
\hline 10 & 18 & 4 & $15-17$ & 1.8 & 28.0 & 70.2 & Silty clay \\
\hline 10 & 18 & 5 & $2-4$ & 3.1 & 12.0 & 84.9 & Clay \\
\hline 11 & 1 & 1 & 7.9 & 9.6 & 8.3 & 82.1 & Clay \\
\hline 11 & 1 & 2 & $14-16$ & 14.2 & 23.5 & 62.3 & Silty clay \\
\hline 11 & 1 & 2 & $87-89$ & 8.5 & 23.2 & 68.3 & Silty clay \\
\hline 11 & 1 & 3 & $14-16$ & 10.2 & 29.5 & 60.3 & Silty clay \\
\hline 11 & 1 & 4 & $14-16$ & 13.4 & 35.3 & 51.3 & Silty clay \\
\hline $11 \mathrm{~A}$ & 4 & 1 & $0-10$ & 1.6 & 65.0 & 33.4 & Clayey silt \\
\hline $11 \mathrm{~A}$ & 4 & 3 & $0-10$ & 2.7 & 82.5 & 14.8 & Silt \\
\hline $11 \mathrm{~A}$ & 4 & 4 & $39-49$ & 1.5 & 51.0 & 47.5 & Clayey silt \\
\hline $12 B$ & 1 & 1 & $32-34$ & 0.0 & 13.2 & 86.8 & Clay \\
\hline $12 \mathrm{~B}$ & 1 & 4 & $135-137$ & 0.1 & 15.2 & 84.7 & Clay \\
\hline $12 \mathrm{~B}$ & 2 & 1 & $128-130$ & 0.4 & 3.6 & 96.0 & Clay \\
\hline $12 \mathrm{~B}$ & 2 & 2 & 16-18 & 0.1 & 6.8 & 93.1 & Clay \\
\hline
\end{tabular}


TABLE 1 - Continued

\begin{tabular}{lccccccl}
\hline Hole & Core & Section & Sampled & $\%$ Sand & $\%$ Silt & \% Clay & Classification \\
\hline $12 \mathrm{~B}$ & 2 & 2 & $45-47$ & 0.0 & 11.0 & 89.0 & Clay \\
$12 \mathrm{C}$ & 1 & 1 & $145-150$ & 11.6 & 55.4 & 33.0 & Clayey silt \\
$12 \mathrm{C}$ & 1 & 2 & $145-150$ & 10.4 & 74.4 & 15.2 & Clayey silt \\
$12 \mathrm{C}$ & 1 & 3 & $145-150$ & 10.2 & 59.4 & 30.4 & Clayey silt \\
$12 \mathrm{C}$ & 1 & 4 & $145-150$ & 8.5 & 37.1 & 54.4 & Silty clay \\
$12 \mathrm{C}$ & $2 \mathrm{R}$ & 1 & 140 & 5.0 & 26.3 & 68.7 & Silty clay \\
$12 \mathrm{C}$ & $2 \mathrm{R}$ & 2 & $145-150$ & 0.5 & 32.8 & 62.7 & Silty clay \\
$12 \mathrm{C}$ & $2 \mathrm{R}$ & 3 & $145-150$ & 0.9 & 17.6 & 81.5 & Clay \\
$12 \mathrm{C}$ & $2 \mathrm{R}$ & 4 & $145-150$ & 4.6 & 18.2 & 77.2 & Clay \\
$12 \mathrm{C}$ & 4 & 1 & $67-69$ & 4.5 & 33.9 & 61.6 & Silty clay \\
$12 \mathrm{C}$ & 4 & 2 & $10-12$ & 0.3 & 26.9 & 72.8 & Silty clay \\
$12 \mathrm{C}$ & 4 & 3 & $1-3$ & 0.1 & 27.5 & 72.4 & Silty clay \\
$12 \mathrm{C}$ & 5 & 2 & $74-76$ & 0.0 & 23.9 & 76.1 & Clay \\
$12 \mathrm{C}$ & 5 & 2 & $10-12$ & 0.4 & 31.1 & 68.5 & Silty clay \\
$12 \mathrm{C}$ & 5 & 3 & $12-14$ & 0.0 & 15.0 & 85.0 & Clay \\
$12 \mathrm{C}$ & 6 & 1 & $110-112$ & 0.0 & 23.0 & 77.0 & Clay \\
$12 \mathrm{C}$ & 6 & 2 & $10-12$ & 0.4 & 19.1 & 80.5 & Clay \\
$12 \mathrm{C}$ & 7 & 1 & 121 & 0.0 & 12.3 & 87.7 & Clay \\
$12 \mathrm{C}$ & 8 & 1 & $75-78$ & 0.1 & 10.9 & 89.0 & Clay \\
$12 \mathrm{C}$ & 9 & 1 & $127-129$ & 0.1 & 6.2 & 93.7 & Clay \\
$12 \mathrm{C}$ & 10 & 1 & $102-104$ & 0.0 & 16.8 & 83.2 & Clay \\
$12 \mathrm{C}$ & 11 & 1 & $56-58$ & 0.0 & 4.7 & 95.3 & Clay \\
\hline
\end{tabular}

TABLE 2

Grain Size Analyses Correlated with Nature of Sediments in Hole 10

\begin{tabular}{|c|c|c|c|c|c|}
\hline Core & Section & $\begin{array}{l}\text { Sampled At } \\
\text { (cm) }\end{array}$ & Classification & Description of Sediment & $\begin{array}{l}\text { Main Constituents of } \\
\text { Coarse Fraction }\end{array}$ \\
\hline 1 & 1 & $60-62$ & Clay & Nannofossil chalk ooze & Foraminifera, fish debris \\
\hline 2 & 2 & $12-14$ & Clay & Nannofossil chalk ooze & Fish debris and ?clinoptilolite \\
\hline 2 & 2 & 129-131 & Silty clay & Mixed zeolite clay and & Fish debris and ?clinoptilolite \\
\hline 2 & 3 & $13-15$ & Silty clay & coccolith ooze & \\
\hline 2 & 3 & $99-101$ & Clay & Calcareous zeolitic red clay & Fish debris and ?clinoptilolite \\
\hline 2 & 4 & $5-7$ & Clay & Nannofossil chalk ooze & Fish debris and ?clinoptilolite \\
\hline 3 & 1 & $35-37$ & Silty clay & Zeolitic nannofossil chalk & Foraminifera, fish debris \\
\hline 3 & 2 & $16-18$ & Silty clay & ooze, Silt varies from 29 & Foraminifera, fish debris \\
\hline 3 & 3 & $10-12$ & Silty clay & 48 per cent depending on & Foraminifera, fish debris \\
\hline 3 & 3 & $81-83$ & Silty clay & coccolith content & Foraminifera, fish debris \\
\hline
\end{tabular}


TABLE 2 - Continued

\begin{tabular}{|c|c|c|c|c|c|}
\hline Core & Section & $\begin{array}{l}\text { Sampled At } \\
(\mathrm{cm})\end{array}$ & Classification & Description of Sediment & $\begin{array}{l}\text { Main Constituents of } \\
\text { Coarse Fraction }\end{array}$ \\
\hline 5 & 1 & $118-120$ & Clay & $\begin{array}{l}\text { Nannofossil chalk ooze, } \\
\text { some zeolite }\end{array}$ & $\begin{array}{l}\text { Foraminifera } \\
\text { Foraminifera }\end{array}$ \\
\hline 5 & 2 & $12-14$ & Silty Clay & Nannofossil ooze & Foraminifera \\
\hline 5 & 2 & $74-76$ & Clay & Nannofossil ooze & Foraminifera, ?clinoptilolite \\
\hline 5 & 3 & $18-20$ & Clayey silt & $\begin{array}{l}\text { Nannofossil chalk ooze, high } \\
\text { silt content due to coccoliths. }\end{array}$ & Foraminifera, \\
\hline 5 & 4,5 & $20-22$ & Silty clay & Cores 5 and 7 are coccolith & Foraminifera, ?clinoptilolite \\
\hline 7 & 1,4 & (See Table 1) & with & chalk ooze with a little zeolite, & Foraminifera, fish debris, ?clinop- \\
\hline 9 & 1,2 & (See Table 1) & clayey silt & Core 9 is a nannofossil radio- & Radiolaria, tilolite \\
\hline 10 & 1,5 & (See Table 1) & & larian chalk ooze, Cores 10,11 & Foraminifera \\
\hline 11 & $1,3,4$ & (See Table 1) & three & and 12 are foraminifera & Foraminifera \\
\hline 12 & 2 & $14-16$ & places & nannofossil chalk ooze. & Foraminifera \\
\hline 12 & 3,4 & (See Table 1) & Clay & Nannofossil foraminifera chalk & Foraminifera \\
\hline 13 & 1 & $120-122$ & Clay & $\begin{array}{l}\text { ooze, with only } 7-10 \text { per cent } \\
\text { silt }\end{array}$ & Foraminifera \\
\hline 13 & 2 & $5-7$ & Silty clay & $\begin{array}{l}\text { Nannofossil foraminifera } \\
\text { chalk ooze }\end{array}$ & Foraminifera \\
\hline 13 & 3,4 & (See Table 1) & Clay & $\begin{array}{l}\text { Nannofossil foraminifera chalk } \\
\text { ooze with some volcanic } \\
\text { minerals }\end{array}$ & Foraminifera \\
\hline 14 & 2,3 & (See Table 1) & Silty clay & Nannofossil foraminifera & Foraminifera \\
\hline 15 & 1,2 & (See Table 1) & Silty clay & chalk ooze with some volcanic & Foraminifera \\
\hline 16 & 1,2 & (See Table 1) & Silty clay & minerals & Foraminifera \\
\hline 16 & 3 & $121-123$ & Clay & $\begin{array}{l}\text { Nannofossil foraminifera chalk } \\
\text { ooze with some dolomite and } \\
\text { zeolite; only } 9 \text { per cent silt }\end{array}$ & Foraminifera \\
\hline 17 & 1,2 & (See Table 1) & Silty clay & $\begin{array}{l}\text { Nannofossil foraminifera chalk } \\
\text { ooze }\end{array}$ & Foraminifera \\
\hline 17 & 3 & $4-6$ & Clay & $\begin{array}{l}\text { Dolomite nannofossil foram- } \\
\text { inifera chalk ooze }\end{array}$ & Foraminifera \\
\hline 18 & $1-4$ & (See Table 1) & Silty clay & $\begin{array}{l}\text { Nannofossil foraminifera chalk } \\
\text { ooze with some dolomite and } \\
\text { volcanic minerals. }\end{array}$ & Foraminifera \\
\hline 18 & 5 & $2-4$ & Clay & $\begin{array}{l}\text { Dolomite nannofossil foram- } \\
\text { inifera chalk ooze }\end{array}$ & Foraminifera \\
\hline
\end{tabular}


TABLE 3

Leg 2 Summary of the Composition of the Coarse Fraction

\begin{tabular}{|c|c|c|c|c|c|c|c|c|c|c|c|c|c|c|c|}
\hline Hole & Core & Section & Foraminifera & Radiolaria & Spicules & $\begin{array}{l}\text { Fish debris } \\
\text { (collophane) }\end{array}$ & \multicolumn{2}{|c|}{ Volcanic glass } & $\begin{array}{c}\text { Siliceous } \\
\text { grains }\end{array}$ & $\begin{array}{l}\text { Carbonate } \\
\text { grains }\end{array}$ & Manganese & $\begin{array}{l}\text { Iron } \\
\text { oxides }\end{array}$ & Clinoptilolite & $\begin{array}{c}\text { Dolomite } \\
\text { (or Rhodochrosite) }\end{array}$ & Others \\
\hline & & & & & & & & & & & & & & & \\
\hline 8 & 1 & 1 & & A & C & & $\mathbf{R}$ & & & & $\mathbf{R}$ & $\mathbf{R}$ & & & R-mica \\
\hline 8 & 2 & $1-3$ & & A & A & & & & & & & & & & \\
\hline $8 \mathrm{~A}$ & 1 & 1 & & A & C & & $\mathbf{R}$ & & & & $\mathbf{R}$ & & & & \\
\hline $8 \mathrm{~A}$ & 1 & 2 & & A & & & & & C & & & & & & \\
\hline 9 & 5 & 1 & $\mathbf{R}$ & A & C & & $\mathbf{R}$ & & & & $\mathbf{R}$ & & & & \\
\hline 9 & 5 & 2 & $\mathbf{R}$ & A & & & & & & $\mathbf{R}$ & $\mathbf{R}$ & & & & \\
\hline 9 & 5 & 3 & $\mathrm{C}$ & $\mathrm{C}$ & & & $\mathbf{R}$ & $\mathbf{R}$ & & A & $\mathbf{R}$ & & & & \\
\hline 9 & 5 & 4 & A & A & & $\mathbf{R}$ & $\mathrm{R}$ & & & & & & & & \\
\hline 9 & 5 & 5 & $\mathbf{R}$ & $\mathbf{R}$ & & & $\mathbf{R}$ & & $\mathbf{R}$ & $\mathbf{R}$ & & & & & \\
\hline 9 & 7 & 1 & C & $\mathrm{C}$ & & A & $\mathbf{R}$ & & & & $\mathbf{R}$ & & & & \\
\hline 9 & 7 & 2 & $\mathbf{R}$ & $\mathrm{C}$ & & $\mathrm{C}$ & $\mathrm{R}$ & & & & C & & & & \\
\hline 9 & 8 & 6 & & & & & & & & & $\mathbf{R}$ & & & A & \\
\hline 9 & 9 & 2 & & $\mathbf{R}$ & & A & $\mathbf{R}$ & & $\mathrm{R}$ & & $\mathbf{R}$ & $\mathbf{R}$ & & & \\
\hline 9 & 9 & 3 & A & & & $\mathrm{C}$ & & $\mathbf{R}$ & & & & $\mathbf{R}$ & & & \\
\hline 9 & 10 & 1 & & & & A & $\mathrm{R}$ & $\mathbf{R}$ & & $\mathbf{R}$ & $\mathbf{R}$ & & & & \\
\hline 9 & 10 & 2 & & $\mathbf{R}$ & & $\mathrm{R}$ & $\mathrm{R}$ & & & & $\mathbf{R}$ & & & & \\
\hline 9 & 10 & 3 & & & & A & $\mathbf{R}$ & $\mathrm{R}$ & & $\mathbf{R}$ & $\mathbf{R}$ & & & & \\
\hline 9 & 10 & $5-6$ & $\mathbf{R}$ & & & $\mathrm{R}$ & $\mathrm{R}$ & & & & $\mathbf{R}$ & & & & A-variety \\
\hline 9 & 12 & 5 & & & $\mathbf{R}$ & $\mathrm{C}$ & C & $\mathrm{C}$ & A & & $\mathrm{R}$ & & & & of ?apatite \\
\hline $9 \mathrm{~A}$ & 1 & 1 & & & & & & & & & $\mathbf{R}$ & & & A & \\
\hline $9 \mathrm{~A}$ & 1 & 5 & & & & $\mathbf{R}$ & & & & & $\mathbf{R}$ & & C & & A-chert \\
\hline $9 \mathrm{~A}$ & 1 & 6 & & & & $\mathbf{R}$ & & & & & $\mathbf{R}$ & & & A & \\
\hline $9 \mathrm{~A}$ & 3 & 1 & & $\mathrm{C}$ & & & $\mathbf{R}$ & & & & & & A & & \\
\hline $9 \mathrm{~A}$ & 3 & 2 & & A & $\mathbf{R}$ & $\mathbf{R}$ & $\mathbf{R}$ & $\mathbf{R}$ & & & & & & C & \\
\hline $9 \mathrm{~A}$ & 3 & 4 & & $\mathbf{R}$ & & & $\mathbf{R}$ & & & & $\mathrm{R}$ & & C & A & \\
\hline 10 & 1 & 2 & $\mathrm{C}$ & & & $\mathrm{C}$ & & & & A & $\mathbf{R}$ & & & & \\
\hline 10 & 2 & 2 & $\mathrm{C}$ & & & $\mathrm{C}$ & & & & $\mathrm{C}$ & $\mathbf{R}$ & & ?C & & \\
\hline 10 & 2 & 3 & $\mathbf{R}$ & & & A & & & & & $\mathbf{R}$ & & ?A & & \\
\hline 10 & 2 & 4 & $\mathrm{C}$ & & & C & & & & & & & ?C & & \\
\hline 10 & 3 & $1-4$ & A & & & $\mathbf{A}$ & $\mathbf{R}$ & $\mathrm{R}$ & & & $\mathrm{R}$ & & ?R & & \\
\hline 10 & 5 & $1-5$ & A & & & C & $\mathbf{R}$ & & & & & & ?C & & \\
\hline 10 & 7 & $1-4$ & $\mathrm{C}$ & & & C & $\mathrm{R}$ & $\mathbf{R}$ & & & & & ?C & & \\
\hline 10 & 9 & $1-2$ & & A & & & & & & & & & & & \\
\hline 10 & 10 & $1-5$ & A & & & $\mathbf{R}$ & & & & & & & & & \\
\hline
\end{tabular}


TABLE 3 - Continued

\begin{tabular}{|c|c|c|c|c|c|c|c|c|c|c|c|c|c|c|c|}
\hline \multirow[b]{2}{*}{ Hole } & \multirow[b]{2}{*}{ Core } & \multirow[b]{2}{*}{ Section } & \multirow[b]{2}{*}{ Foraminifera } & \multirow[b]{2}{*}{ Radiolaria } & \multirow[b]{2}{*}{ Spicules } & \multirow{2}{*}{$\begin{array}{l}\text { Fish debris } \\
\text { (collophane) }\end{array}$} & \multicolumn{2}{|c|}{ Volcanic glass } & \multirow{2}{*}{$\begin{array}{l}\text { Siliceous } \\
\text { grains }\end{array}$} & \multirow{2}{*}{$\begin{array}{c}\text { Carbonate } \\
\text { grains }\end{array}$} & \multirow[b]{2}{*}{ Manganese } & \multirow{2}{*}{$\begin{array}{l}\text { Iron } \\
\text { oxides }\end{array}$} & \multirow[b]{2}{*}{ Clinoptilolite } & \multirow{2}{*}{$\begin{array}{l}\text { Dolomite } \\
\text { (or Rhodochrosite) }\end{array}$} & \multirow[b]{2}{*}{ Others } \\
\hline & & & & & & & clear & yellow & & & & & & & \\
\hline 10 & 11 & $1-4$ & A & $\mathbf{R}$ & & & & & & & $\mathrm{R}$ & & & & \\
\hline 10 & 12 & $2-4$ & A & & & $\mathbf{R}$ & $\mathbf{R}$ & & & & & & & & \\
\hline 10 & 13 & $1-4$ & A & & & $\mathbf{R}$ & & & & & $\mathbf{R}$ & & & & \\
\hline 10 & 14 & $2-3$ & A & & & & & & & $\mathbf{R}$ & $\mathbf{R}$ & & & & \\
\hline 10 & 15 & $1-2$ & A & & & & & & & - & & & & & \\
\hline 10 & 16 & $1-3$ & A & & & & $\mathbf{R}$ & & & & & & & & \\
\hline 10 & 17 & $1-3$ & A & & & & & & & & $\mathrm{R}$ & $\mathrm{R}$ & & & \\
\hline 10 & 18 & $1-5$ & $\mathbf{A}$ & & & & & & & & $\mathbf{R}$ & & & & \\
\hline 11 & 1 & $1-3$ & $\mathbf{A}$ & & & & & & & & $\mathbf{R}$ & & & & \\
\hline $11 \mathrm{~A}$ & 4 & $1-4$ & $\mathbf{A}$ & & & & & & & & $\mathbf{R}$ & $\mathbf{R}$ & & & \\
\hline $12 B$ & 1 & 4 & & & & & A & $\mathrm{R}$ & & & & & & & \\
\hline $12 B$ & 2 & 2 & & $\mathrm{R}$ & & & $\mathbf{R}$ & $\mathbf{R}$ & & & A & & & & \\
\hline $12 \mathrm{C}$ & 1 & $\begin{array}{c}1,2 \mathrm{R} \\
4\end{array}$ & A & & & $\mathbf{R}$ & $\mathbf{R}$ & & & & $\mathbf{R}$ & & & & \\
\hline $12 \mathrm{C}$ & 5 & 2,3 & & & & $\mathrm{C}$ & $\mathbf{R}$ & & & & A & & ?A & & \\
\hline $12 \mathrm{C}$ & 6 & $1-2$ & & & & $\mathbf{R}$ & C & & & & A & $\mathbf{R}$ & ?R & & \\
\hline $12 \mathrm{C}$ & 8 & 1 & & & & A & $\mathrm{C}$ & A & & & $\mathrm{R}$ & $\mathrm{R}$ & & & \\
\hline $12 \mathrm{C}$ & 9 & 1 & & & & A & $\mathrm{C}$ & $\mathbf{R}$ & & & & & ? R & & \\
\hline
\end{tabular}

